of Geomagnetic Phenomena appeared in 1967. Solid-Earth geomagnetism had expanded, but then so had its external counterpart, aeronomy, largely as a result of the advent of rockets and satellites.

If the two branches of the subject must be dealt with together, there is now clearly a case for giving solid-Earth geomagnetism a fairer share of the proceedings and that is what Jacobs evidently intends that Geomagnetism, a new multi-authored work, should do. It was envisaged that the two volumes published so far would completely cover the solid-Earth aspects of geomagnetism and that they would be followed (in about a year's time) by two further volumes devoted exclusively to aeronomy - a convenient split. Unfortunately, for reasons too complex to be explained here, some of the solid-Earth topics have had to be deferred. Those interested solely in the main field will therefore later have to beg, borrow or steal at least one other volume containing much material of little concern to them; but they will hardly be able to complain that the balance of topics has not been handsomely redressed in their favour.

In the first two volumes, then, ten specialists cover the history of geomagnetic observation, instrumentation in general, instrumentation and experimental methods for oceanic observation, the main geomagnetic field and its changes, the crustal field, magnetohydrodynamics of the Earth's core, the dynamics and kinematics of the origin of the main field (two separate chapters), lunar palaeomagnetism, planetary magnetism, and the magnetism of meteorites. The topics to come are magnetotellurics, terrestrial palaeomagnetism and lunar magnetism. Although etymological purists may object to the trend, it is perhaps worth emphasizing that 'geomagnetism' now includes the magnetism of extraterrestrial bodies. Indeed, over the past two decades or so, beyond-Earth studies have been the subject's chief growth area.

Because any one of the above-mentioned topics could make a two-volume set in itself, comprehensive treatment within a single chapter is hardly to be expected, although some chapters (especially that on the main field) manage to pack in a surprising amount of detail. Nevertheless, all of the contributions provide good, upto-date general surveys of their topics (albeit only for the mathematically proficient) and will thus prove useful starting points for the new postgraduate at whom they are presumably aimed. Chapman and Bartels were able to produce a detailed treatise on all existing knowledge of the subject. Forty-eight years of work later, such luxury is regrettably no longer feasible, even with a large team of writers.

Peter J. Smith is a Reader in the Department of Earth Sciences, Open University, Walton Hall, Milton Keynes MK7 6AA, UK.

\title{
Mutating molecules
}

\section{N.H. Barton and J.S. Jones}

Molecular Evolutionary Genetics. By Masatoshi Nei. Columbia University Press: 1987. Pp. 512. \$50, £29.50.

IN population genetics, the theoretical tail has usually wagged the experimental dog. At a time when mathematicians were producing elegant models of evolutionary change, biologists were still struggling with problems which seemed insoluble: how much genetic variation is there, what is the strength of natural selection and what genetic changes are involved in the formation of new species?

However, the startling new discoveries of molecular biology - homology between distantly related organisms, quantities of apparently functionless DNA, widespread gene duplication which may lead to volcanic eruptions of pseudogenes from those which still retain a function, and the concerted differentiation of repeated sequences - all now demand explanation by population genetics theory. Even the discrete nature of the gene looks increasingly foggy, and pea soup, rather than peas, is now the geneticist's staple diet. As Nei points out, there is an urgent need for molecular biology and population genetics to be unified into 'molecular evolutionary genetics'. His book attempts to do that, and provides a valuable compendium of up-to-date molecular information and of the statistical methods by which it can be described.

After an account of the evolutionary history of life and of the remarkably good general fit between the amino acid sequences of particular proteins in different species and their dates of divergence, Nei goes on to the mass of new data on the evolution of nucleotide sequences. Just as with proteins, rates of evolution at the DNA level seem to depend greatly on 'functional constraints'. Some sequences have scarcely changed during the history of life, presumably because purifying selection removes any mutations which deviate from the functional norm. In other cases, however, related species may diverge very rapidly in DNA sequence, a pattern usually interpreted as a result of the relaxation of selection when no functional transcript is produced.

This reflects a remarkable difference in emphasis between molecular and morphological evolution. Before the advent of molecular biology, rapid change was seen as a proof of natural selection, whereas it is now generally taken as evidence against it. In the same way, molecular evolution has it that nature abhors a polymorphism; genetic variation once thought to demand an explanation in terms of balancing selection is now seen simply as the passage of a transient neutral variant through a population. As is so often true in evolutionary biology, these apparent contradictions may be due largely to differences in the timescales involved: neutral sequence divergence takes place over millions of years, whereas selection can cause drastic phenotypic changes in a few generations.

Nei goes on to give an account of a variety of sequence alignment and treebuilding methods which can introduce molecular biologists to evolutionary data that they never knew they had. Molecular information is invaluable in providing an objective basis for the phylogenetic classifications which are at the centre of much of biology. He ends with a personal view of the "new evolutionary biology' that may be needed to cope with modern molecular genetics. Somewhat surprisingly, this turns out to be close to the theories of evolutionary genetics which first emerged from the rediscovery of Mendelism at the turn of the century. Nei argues that mutation is the creative process at both the molecular and the phenotypic level: natural selection is primarily conservative, and simply weeds out the unfit. His point that "natural selection does not create any new genotypes" is reminiscent of arguments such as that of Johanssen in 1915 that "selection of differing individuals creates nothing new". This emphasis on the importance of mutation can be misleading: selection and mutation work together in a long process of trial and error which can build up adapted genotypes that would stand almost no chance of assembly by random mutation alone.

It is particularly hard to see how mutation could shape adaptations if these involve the gradual accumulation of many genetic changes of small individual effect. Nei argues that most adaptations involve just a few major mutations. Although the drastic morphological changes induced by homoeotic and heterochronic mutations support this idea, the discovery that gene regulation can depend on a diffuse set of sequences, well outside the coding region, points in the opposite direction.

Nei's bold conclusions sit slightly oddly with the inevitable uncertainties involved in explaining the torrent of new molecular information: indeed, it may take as long to reach a proper understanding of the natural history of molecules as it has to elucidate that of whole organisms. Nei's book gives a remarkably complete and upto-date account of the present state of the relationship between theory and practice in molecular evolution. But we should not be surprised if his second edition turns out to be very different from the first.

N.H. Barton and J.S. Jones are in the Department of Genetics and Biometry, University College London, 4 Stephenson Way, London NW1 $2 H E, U K$. 